\title{
OVERDETERMINED PROBLEMS FOR THE NORMALIZED $p$-LAPLACIAN
}

\author{
AGNID BANERJEE AND BERND KAWOHL \\ (Communicated by Joachim Krieger)
}

\begin{abstract}
We extend the symmetry result of Serrin [21] and Weinberger 24 from the Laplacian operator to the highly degenerate game-theoretic $p$ Laplacian operator and show that viscosity solutions of $-\Delta_{p}^{N} u=1$ in $\Omega, u=0$ and $\frac{\partial u}{\partial \nu}=-c \neq 0$ on $\partial \Omega$ can only exist on a bounded domain $\Omega$ if $\Omega$ is a ball.
\end{abstract}

\section{INTRODUCTION}

In a seminal paper 21 Serrin showed that the following overdetermined boundary problem can only have a solution $u \in C^{2}(\bar{\Omega})$ if $\Omega$ is a ball.

$$
-\Delta u=1 \quad \text { in } \Omega, \quad u=0 \quad \text { and } \quad \frac{\partial u}{\partial \nu}=c<0 \text { on } \partial \Omega .
$$

Here $c$ is constant and $\Omega \subset \mathbb{R}^{n}$ is a bounded connected domain with boundary of class $C^{2}$. Serrin used Alexandrov's moving plane method for his proof, while Weinberger 24] found a proof using Rellich's identity and the fact that a related function $P(x)=|\nabla u|^{2}+\frac{2}{n} u$ is constant in $\Omega$. Only the second method of proof has been adapted to a situation where the Laplacian operator is replaced by the $p$-Laplacian in [9] and [13].

In this paper we treat the case that the Laplacian is replaced by the normalized or game-theoretic $p$-Laplacian $\Delta_{p}^{N}$ which is defined for any $p \in(1, \infty)$ by

$$
\Delta_{p}^{N} u:=\frac{1}{p}|\nabla u|^{2-p} \operatorname{div}\left(|\nabla u|^{p-2} \nabla u\right)=\frac{1}{p} \Delta_{1}^{N} u+\frac{p-1}{p} \Delta_{\infty}^{N} u,
$$

a convex combination of the limiting operators

$$
\Delta_{1}^{N} u:=|\nabla u| \operatorname{div}\left(\frac{\nabla u}{|\nabla u|}\right) \quad \text { and } \quad \Delta_{\infty}^{N} u:=\frac{\sum_{i, j=1}^{n} u_{x_{i}} u_{x_{i} x_{j}} u_{x_{j}}}{|\nabla u|^{2}} .
$$

Note that this operator is not in divergence form. Therefore it resists attempts to treat it with variational methods. On the other hand it is quite benign, because its coefficient matrix is bounded from below by $\min \left\{\frac{1}{p}, \frac{p-1}{p}\right\} I$ and from above by $\max \left\{\frac{1}{p}, \frac{p-1}{p}\right\} I$. Therefore the moving plane method seems more appropriate in this context.

Note also, that the above definition of the normalized $p$-Laplacian needs further explanation when $\nabla u=0$. The definition of and a weak comparison principle

Received by the editors November 23, 2017, and, in revised form, January 5, 2018.

2010 Mathematics Subject Classification. Primary 35N25, 36J62, 35D40.

Key words and phrases. Overdetermined boundary value problem, game-theoretic $p$ Laplacian, viscosity solution.

(C)2018 by the authors under Creative Commons Attribution-Noncommercial 3.0 License (CC BY NC 3.0) 
for continuous viscosity solutions are given below. These and an existence and uniqueness result can be found for instance in [18] or [17. Our main result answers an open problem from [14.

Theorem 1.1. For $p \in(1, \infty)$ let $u \in C(\bar{\Omega})$ be a viscosity solution to the overdetermined boundary value problem

$$
-\Delta_{p}^{N} u=1 \quad \text { in } \Omega, \quad u=0 \quad \text { and } \quad \frac{\partial u}{\partial \nu}=c<0 \text { on } \partial \Omega
$$

on a connected bounded domain $\Omega$ with boundary of class $C^{2}$. Then $\Omega$ must be a ball.

Remark 1.2. We note that the Neumann condition $\frac{\partial u}{\partial \nu}=c<0$ on $\partial \Omega$ is interpreted in the following sense: Any $C^{2}$ function $\varphi$ such that $\varphi-u$ has a minimum at a point $x \in \partial \Omega$ satisfies $\frac{\partial \varphi}{\partial \nu}(x) \leq c$ at $x$. Similarly, any $C^{2}$ function $\psi$ such that $\psi-u$ has a maximum at a point $y \in \partial \Omega$ satisfies $\frac{\partial \psi}{\partial \nu}(y) \geq c$.

Remark 1.3. It was pointed out in 14 that Theorem 1.1 remains true for $p=1$, while for $p=\infty$ it is generally false.

In fact for $p=1$ the equation can be rewritten as $-\frac{n-1}{p} H(x) u_{\nu}(x)=1$, where $H(x)$ denotes mean curvature of the level set passing through $x$, and in view of the constant Neumann data this means that $\partial \Omega$ has constant mean curvature. Therefore $\Omega$ is a ball of radius $\frac{1-n}{p} c$.

As explained in [5], for $p=\infty$ the right $P$-function is $|\nabla u|^{2}+2 u$, and annuli are cases in which the overdetermined problem has viscosity solutions of class $C^{1}$. The case $p=\infty$ was also studied in great detail in a series of papers by Crasta and Fragalá, who relaxed the $C^{2}$ smoothness of the boundary, see e.g. [7].

The normalized $p$-Laplacian has also been studied in the context of evolution equations in a number of papers, see $[11,8,4,3,20,12,10$.

\section{Definitions and Comparison Result}

In the notation of the theory of viscosity solutions we study the equation

$$
F_{p}\left(\nabla u, \nabla^{2} u\right)=-\frac{p-2}{2}|\nabla u|^{-2}\left\langle\nabla^{2} u \nabla u, \nabla u\right\rangle-\frac{1}{p} \operatorname{trace} \nabla^{2} u-1=0 .
$$

Definition 2.1. Following [6], $u \in C(\Omega)$ is a viscosity solution of the equation $F\left(\nabla u, \nabla^{2} u\right)=0$, if it is both a viscosity subsolution and a viscosity supersolution. $u$ is a viscosity subsolution of $F\left(\nabla u, \nabla^{2} u\right)=0$, if for every $x \in \Omega$ and $\varphi \in C^{2}$ such that $\varphi-u$ has a minimum at $x$, the inequality $F_{*}\left(\nabla \varphi, \nabla^{2} \varphi\right) \leq 0$ holds. Here $F_{*}$ is the lower semicontinuous hull of $F$.

$u$ is a viscosity supersolution of $F\left(\nabla u, \nabla^{2} u\right)=0$, if for every $x \in \Omega$ and $\psi \in C^{2}$ such that $\psi-u$ has a maximum at $x$, the inequality $F^{*}\left(\nabla \psi, \nabla^{2} \psi\right) \geq 0$ holds. Here $F^{*}$ is the upper semicontinuous hull of $F$.

If $X$ denotes a symmetric real valued matrix, we denote its eigenvalues by $\lambda_{\min }=$ $\lambda_{1} \leq \lambda_{2} \leq \ldots \leq \lambda_{n}=\lambda_{\max }$. Using this notation, it is a simple exercise to find out that

$$
F_{*}(q, X)=\left\{\begin{array}{cl}
F(q, X) & \text { if } q \neq 0, \\
\inf _{a \in \mathbb{R}^{n} \backslash\{0\}} F(a, X) & \text { if } q=0,
\end{array}\right.
$$


SO

$$
F_{*}(0, X)=\left\{\begin{array}{lc}
-\frac{p-1}{p} \lambda_{1}-\frac{1}{p} \sum_{i=2}^{n} \lambda_{i}-1 & \text { for } p \in(1,2], \\
-\frac{p-1}{p} \lambda_{n}-\frac{1}{p} \sum_{i=1}^{n-1} \lambda_{i}-1 & \text { for } p \in[2, \infty)
\end{array}\right.
$$

while

$$
F^{*}(q, X)=\left\{\begin{array}{cl}
F(q, X) & \text { if } q \neq 0, \\
\sup _{a \in \mathbb{R}^{n} \backslash\{0\}} F(a, X) & \text { if } q=0,
\end{array}\right.
$$

that is

$$
F^{*}(0, X)=\left\{\begin{array}{lc}
-\frac{p-1}{p} \lambda_{n}-\frac{1}{p} \sum_{i=1}^{n-1} \lambda_{i}-1 & \text { for } p \in(1,2], \\
-\frac{p-1}{p} \lambda_{1}-\frac{1}{p} \sum_{i=2}^{n} \lambda_{i}-1 & \text { for } p \in[2, \infty) .
\end{array}\right.
$$

The following comparison principle has been derived in [18, 17].

Proposition 2.2. Suppose $u$ and $v$ are in $C(\bar{D})$ and are viscosity super- resp. subsolutions of $F_{p}=0$ on a domain $D$ and $u \geq v$ on $\partial D$. Then $u \geq v$ in $D$.

It can be used to show the positivity of $u$ and a Hopf Lemma.

Lemma 2.3. Suppose $\Omega$ satisfies a uniform interior sphere condition and $u \in C(\bar{\Omega})$ is a viscosity solution of $F_{p}\left(\nabla u, \nabla^{2} u\right)=0$ in $\Omega$ such that $u=0$ on $\partial \Omega$. Then $u$ is positive in $\Omega$ and there exists a number $a>0$ such that for all $y \in \partial \Omega$

$$
\limsup _{t \rightarrow 0^{+}} \frac{u(y)-u(y-t \nu(y))}{t} \leq-a<0 .
$$

Here $\nu(y)$ denotes the outward unit normal at $y$. In fact, one can compare $u$ to a radially symmetric and radially decreasing solution $v$ on the interior of the sphere. On a ball solutions $v$ of the Dirichlet problem for $F_{p}=0$ are unique by Proposition 2.2, so they are necessarily radial. In polar coordinates $F_{p}=0$ turns into the tractable ODE 15

$$
-\frac{p-1}{p} v_{r r}-\frac{n-1}{p r} v_{r}=1 \quad \text { in }(0, R)
$$

with $v_{r}(0)=0=v(R)$ as boundary conditions, and this boundary value problem has the explicit solution

$$
v(r)=\frac{p}{2(p+n-2)}\left(R^{2}-r^{2}\right)
$$

so that $u$ is positive in every ball with radius $R$ contained in $\Omega$. Moreover, Lemma 2.3 holds with $a=\frac{R p}{p+n-2}$.

\section{Proof of Main Result}

To prove Theorem 1.1 we follow an idea developed in [1. We first note that from the regularity result stated in Theorem 4.2 in the Appendix, we have that $u$ is in $C^{1, \beta}(\bar{\Omega})$ for some $\beta=\beta(n, p, \Omega)$ and therefore the Neumann condition is realized in the classical pointwise sense. Now because by assumption $|\nabla u|=-c>0$ on $\partial \Omega$ and $u \in C^{1, \beta}(\bar{\Omega})$, we have that $|\nabla u|>0$ in an $\varepsilon$-neighborhood $S_{\varepsilon}$ of $\partial \Omega$ inside $\Omega$ defined by $S_{\varepsilon}:=\{x \in \bar{\Omega} ; d(x, \partial \Omega)<\varepsilon\}$. Therefore the operator $F_{p}$ is well-defined in the classical sense in $S_{\varepsilon}$. Moreover in $S_{\varepsilon}$, since $|\nabla u|>0$, we have that $u$ solves

$$
\sum_{i, j=1}^{n} a_{i j}(x) u_{x_{i} x_{j}}=-1
$$


where

$$
a_{i j}(x)=\frac{1}{p}\left(\delta_{i j}+(p-2) \frac{u_{x_{i}} u_{x_{j}}}{|\nabla u|^{2}}\right)
$$

is uniformly elliptic and is in $C^{\beta}\left(S_{\varepsilon}\right)$. Consequently, by the classical Schauder theory we can assert that $u$ is of class $C_{l o c}^{2, \beta}$ in $S_{\varepsilon}$. We now move a hyperplane, say $T_{\lambda}:=\left\{x \in \mathbb{R}^{n} \mid x_{1}=\lambda\right\}$ from the left by the amount $\varepsilon / 2$ in $x_{1}$-direction into $\Omega$ and compare the original solution $u(x)$ to the reflected one $v(x)=u\left(x-2 \lambda e_{1}\right)$ in the reflected cap. By the weak comparison principle, Proposition 2.2 , we know that $u \geq v$ in the reflected cap $\Sigma_{\lambda}^{\prime}$. Moreover, since $|\nabla u|,|\nabla v|>0$ in $S_{\varepsilon}$, we have that $u, v$ solve an equation in $S_{\varepsilon}$ of the form

$$
\tilde{F}\left(\nabla h, \nabla^{2} h\right)=0
$$

where $\tilde{F}$ is uniformly elliptic and smooth in its arguments. Therefore $w=u-v$ solves the following linearized equation in $S_{\varepsilon}$,

$$
\sum_{i, j=1}^{n} c_{i j} w_{x_{i} x_{j}}+\langle b, \nabla w\rangle=0,
$$

where

and

$$
c_{i j}=\int_{0}^{1} \frac{\partial \tilde{F}}{\partial m_{i j}}\left(t \nabla u+(1-t) \nabla v, t \nabla^{2} u+(1-t) \nabla^{2} v\right) d t
$$

$$
b_{i}=\int_{0}^{1} \frac{\partial \tilde{F}}{\partial p_{i}}\left(t \nabla u+(1-t) \nabla v, t \nabla^{2} u+(1-t) \nabla^{2} v\right) d t .
$$

Moreover $\left[c_{i j}\right]$ is uniformly elliptic and the first order coefficients $b_{i}$ are bounded in $S_{\varepsilon}$. Note that over here, we think of $\tilde{F}: \mathbb{R}^{n} \times \mathbb{R}^{n^{2}} \rightarrow \mathbb{R}$ as a function of the matrix $\left[m_{i j}\right] \in \mathbb{R}^{n^{2}}$ and the vector $p=\left(p_{1}, \ldots . ., p_{n}\right) \in \mathbb{R}^{n}$. Therefore, $\frac{\partial \tilde{F}}{\partial m_{i j}}$ is to be thought of as the partial derivative of $\tilde{F}$ with respect to the coordinate $m_{i j}$ in $\mathbb{R}^{n^{2}}$ and $\frac{\partial \tilde{F}}{\partial p_{i}}$ is the partial derivative with respect to the coordinate $p_{i}$ in $\mathbb{R}^{n}$.

Since $w$ solves the uniformly elliptic PDE (3.2) in $\Sigma_{\lambda}^{\prime} \cap S_{\varepsilon}$, by the classical strong maximum principle applied to $w$, we get that $u>v$ in $\Sigma_{\lambda}^{\prime} \cap S_{\varepsilon}$ and $w_{x_{1}}>0$ on the plane $T_{\lambda} \cap S_{\varepsilon / 2}$. The latter inequality follows from the classical Hopf Lemma applied to $w$. We continue to move the hyperplane across. Even if the operator might become degenerate because we pass a critical point of $u$, the weak comparison principle continues to hold, so that $u \geq v$ in the reflected cap, until one of the following cases occurs.

i) The hyperplane and $\partial \Omega$ meet under a right angle in a point $P$.

ii) The reflected cap touches $\partial \Omega$ from the inside of $\Omega$ in a point $Q$.

In case i) we can apply the strong maximum principle again and conclude that either $u>v$ in the reflected cap intersected with an $\varepsilon / 2$ neighborhood of the point $P$, or $u \equiv v$ there. But by Serrin's corner lemma applied to $w=u-v$ which solves (3.2), the first case $w>0$ is ruled out. In fact $\nabla u(P)=\nabla v(P)$ because the normal derivatives coincide there and the tangential ones vanish. So a partial derivative of $w$ in any direction $\eta$ must vanish there. Let us see what happens to second partial derivatives in direction $\eta=\alpha \nu+\beta \tau$, where $\tau$ is a unit vector tangent to $\partial \Omega$ at $P$. We claim that

$$
u_{\eta \eta}(P)=\alpha^{2} u_{\nu \nu}(P)+\alpha \beta u_{\nu \tau}(P)+\alpha \beta u_{\tau \nu}(P)+\beta^{2} u_{\tau \tau}(P)=v_{\eta \eta}(P) .
$$

In fact in $P$ one can rewrite the differential equations for $u$ and $v$ as (see [14])

$$
\frac{p-1}{p} u_{\nu \nu}(P)+\frac{n-1}{p} H(P) u_{\nu}(P)=-1=\frac{p-1}{p} v_{\nu \nu}(P)+\frac{n-1}{p} H(P) v_{\nu}(P)
$$


where $H$ is the mean curvature of the $C^{2}$ boundary. Since $u_{\nu}(P)=c=v_{\nu}(P)$, we conclude that $u_{\nu \nu}(P)=v_{\nu \nu}(P)$. For the same reason, $\left(u_{\nu}\right)_{\tau}=0=\left(v_{\nu}\right)_{\tau}$. Now $\left(u_{\tau}\right)_{\nu}=u_{\nu \tau}-\kappa u_{\nu}$, where $\kappa$ denotes the curvature of $\partial \Omega$ in direction $\tau$, so that $\left(u_{\tau}\right)_{\nu}(P)=\left(v_{\tau}\right)_{\nu}(P)$. Finally one can observe that $u_{\tau \tau}(P)=u_{s s}(P)+\kappa(P) u_{\nu}(P)$, where $s$ denotes arclength along the curve that is cut out of $\partial \Omega$ by the plane spanned by $\tau$ and $\nu$. Since $u$ and $v$ are constant on $\partial \Omega$, we have $u_{s s}(P)=v_{s s}(P)$, and since $\nabla u(P)=\nabla v(P)$, this completes the proof of (3.3). Therefore we can conclude that $w_{\eta}(P)=w_{\eta \eta}(P)=0$ and at this point, Serrin's corner lemma implies that $w \equiv 0$ in $\Sigma_{\lambda}^{\prime} \cap S_{\varepsilon}$.

In case ii) we can also conclude that either $u>v$ in the reflected cap intersected with an $\varepsilon / 2$ neighborhood of the point $Q$ or $u \equiv v$. Now in the former case, i.e., when $u>v$ in the reflected cap, we recall again that $w$ solves the uniformly elliptic PDE (3.2) in $S_{\varepsilon}$, but then by Hopf's Lemma applied to $w$, we get that $\partial w / \partial \nu<0$ at $Q$, which contradicts the fact that $u$ satisfies constant Neumann data. Consequently, we have that $w \equiv 0$ in $\Sigma_{\lambda}^{\prime} \cap S_{\varepsilon}$.

In both cases $\partial \Omega$ and $u$ are locally Steiner-symmetric in direction $x_{1}$. To see that they are also globally symmetric, one can argue as follows. For reasons of continuity the set of points in which the reflected boundary coincides with the original boundary is closed in $\partial \Omega$. But it is also open in $\partial \Omega$. In fact in any point that belongs to the boundary of this intersection one can apply the corner lemma again to see that a whole neighborhood still belongs to it.

Since this Steiner symmetry happens in any direction, we can conclude that $\Omega$ is a ball and $u$ is radial and radially decreasing.

\section{Appendix}

In this section, we state and prove a basic regularity result which has been referred to in the proof of Theorem 1.1 in the previous section. In order to do so, we first introduce the relevant notion of extremal Pucci type operators. Let $\mathcal{M}^{+}$ and $\mathcal{M}^{-}$denote the maximal and minimal Pucci operators corresponding to $\lambda, \Lambda$, i.e., for every $M \in \mathcal{S}_{n}$ we have

$$
\begin{aligned}
& \mathcal{M}^{+}(M)=\mathcal{M}^{+}(M, \lambda, \Lambda)=\Lambda \sum_{e_{i}>0} e_{i}+\lambda \sum_{e_{i}<0} e_{i}, \\
& \mathcal{M}^{-}(M)=\mathcal{M}^{-}(M, \lambda, \Lambda)=\lambda \sum_{e_{i}>0} e_{i}+\Lambda \sum_{e_{i}<0} e_{i},
\end{aligned}
$$

where $e_{i}=e_{i}(M)$ indicate the eigenvalues of $M$. Hereafter, the dependence of $\mathcal{M}^{+}$ and $\mathcal{M}^{-}$on $\lambda, \Lambda$ will be suppressed. As is well known, $\mathcal{M}^{+}$and $\mathcal{M}^{-}$are uniformly elliptic fully nonlinear operators.

We now state the following important lemma which connects the normalized $p$ Laplacian operator $\Delta_{p}^{N}$ to appropriate maximal and minimal Pucci operators.

Lemma 4.1. Let $u \in C(\Omega)$ be a viscosity solution to

$$
\Delta_{p}^{N} u=f \text { in } \Omega
$$

and let $f$ be bounded. Then $u$ satisfies the following differential inequalities in the viscosity sense

$$
\mathcal{M}^{+}\left(\nabla^{2} u\right)+K \geq 0 \geq \mathcal{M}^{-}\left(\nabla^{2} u\right)-K
$$


where $\mathcal{M}^{+}, \mathcal{M}^{-}$are the pair of extremal Pucci operators corresponding to $\lambda=$ $\min \left\{\frac{1}{p}, \frac{p-1}{p}\right\}$ and $\Lambda=\max \left\{\frac{1}{p}, \frac{p-1}{p}\right\}$ and $K=\|f\|_{L^{\infty}(\Omega)}$.

Proof. The proof is a straightforward consequence of the fact that the coefficient matrix corresponding to $\Delta_{p}^{N}$ is bounded from below by $\min \left\{\frac{1}{p}, \frac{p-1}{p}\right\} I$ and from above by $\max \left\{\frac{1}{p}, \frac{p-1}{p}\right\} I$.

We now state our main result in this section concerning the regularity of viscosity solutions to the equation in Theorem 1.1 up to the boundary.

Theorem 4.2. Let $\Omega$ be a $C^{2}$ domain and $x_{0} \in \partial \Omega$. Let $u$ be a viscosity solution to

$$
\left\{\begin{array}{l}
-\Delta_{p}^{N} u=f \text { in } \Omega \cap B_{2 r}\left(x_{0}\right) \\
u=g \text { on } \partial \Omega \cap B_{2 r}\left(x_{0}\right)
\end{array}\right.
$$

where $f \in C\left(\overline{B_{2 r}\left(x_{0}\right) \cap \Omega}\right)$ and $g \in C^{1, \alpha}\left(\partial \Omega \cap B_{2 r}\left(x_{0}\right)\right)$. Then $u \in C^{1, \beta}\left(\overline{\Omega \cap B_{r}\left(x_{0}\right)}\right)$ where $\beta=\beta(\alpha, \Omega, f, g)$.

Proof. We first note that by Lemma 4.1, $u$ satisfies the differential inequalities (4.2) in the viscosity sense. Therefore we may apply Theorem 1.1 in 22 and can assert that for some $\beta=\beta(\alpha, n, p)$, there exists $G \in C^{\beta_{1}}\left(\overline{\partial \Omega \cap B_{r}\left(x_{0}\right)}\right)$ which is the "gradient" of $u$ at the boundary such that

$$
\left|u(x)-u\left(x_{1}\right)-\left\langle G\left(x_{1}\right), x-x_{1}\right\rangle\right| \leq C\left|x-x_{1}\right|^{1+\beta_{1}} \text { for all } x \in \Omega \cap B_{r}\left(x_{0}\right) .
$$

Here $C$ depends also on the $C^{2}$ character of the domain $\Omega$. Therefore, (4.4) expresses the fact that $u$ is $C^{1, \beta}$ at the boundary. Now the fact that $u \in C_{l o c}^{1, \beta_{2}}\left(\Omega \cap B_{2 r}\left(x_{0}\right)\right)$ for some $\beta_{2}$ depending on $n, p$ follows from the interior regularity result established in the recent paper [2]. At this point, by taking $\beta=\min \left(\beta_{1}, \beta_{2}\right)$, we can argue as in the proof of Proposition 2.4 in $\left[19\right.$ to conclude that $u \in C^{1, \beta}\left(\overline{\Omega \cap B_{r}\left(x_{0}\right)}\right)$.

\section{ACKnowledgments}

This research was essentially done during a visit of the second author to TIFR CAM Bangalore. The second author thanks the institute and in particular Agnid Banerjee for their hospitality and support. Both authors thank the anonymous referee for carefully reading the manuscript and for his/her helpful comments and suggestions.

\section{References}

[1] Giovanni Alessandrini and Nicola Garofalo, Symmetry for degenerate parabolic equations, Arch. Rational Mech. Anal. 108 (1989), no. 2, 161-174. MR 1011556

[2] Amal Attouchi, Mikko Parviainen, and Eero Ruosteenoja, $C^{\overline{1, \alpha}}$ regularity for the normalized p-Poisson problem (English, with English and French summaries), J. Math. Pures Appl. (9) 108 (2017), no. 4, 553-591. MR 3698169

[3] Agnid Banerjee and Nicola Garofalo, Gradient bounds and monotonicity of the energy for some nonlinear singular diffusion equations, Indiana Univ. Math. J. 62 (2013), no. 2, 699736. MR 3158523

[4] Agnid Banerjee and Nicola Garofalo, On the Dirichlet boundary value problem for the normalized p-Laplacian evolution, Commun. Pure Appl. Anal. 14 (2015), no. 1, 1-21. MR.3299022

[5] Giuseppe Buttazzo and Bernd Kawohl, Overdetermined boundary value problems for the $\infty$-Laplacian, Int. Math. Res. Not. IMRN 2 (2011), 237-247. MR2764863 
[6] Michael G. Crandall, Hitoshi Ishii, and Pierre-Louis Lions, User's guide to viscosity solutions of second order partial differential equations, Bull. Amer. Math. Soc. (N.S.) 27 (1992), no. 1, 1-67. MR 1118699

[7] Graziano Crasta and Ilaria Fragalà, Characterization of stadium-like domains via boundary value problems for the infinity Laplacian, Nonlinear Anal. 133 (2016), 228-249. MR3449756

[8] Kerstin Does, An evolution equation involving the normalized p-Laplacian, Commun. Pure Appl. Anal. 10 (2011), no. 1, 361-396. MR2746543

[9] Nicola Garofalo and John L. Lewis, A symmetry result related to some overdetermined boundary value problems, Amer. J. Math. 111 (1989), no. 1, 9-33. MR.980297

[10] Tianling Jin and Luis Silvestre, Hölder gradient estimates for parabolic homogeneous $p$ Laplacian equations (English, with English and French summaries), J. Math. Pures Appl. (9) 108 (2017), no. 1, 63-87. MR3660769

[11] Petri Juutinen and Bernd Kawohl, On the evolution governed by the infinity Laplacian, Math. Ann. 335 (2006), no. 4, 819-851. MR2232018

[12] Petri Juutinen, Decay estimates in the supremum norm for the solutions to a nonlinear evolution equation, Proc. Roy. Soc. Edinburgh Sect. A 144 (2014), no. 3, 557-566. MR3207437

[13] B. Kawohl, Overdetermined problems and the p-Laplacian, Acta Math. Univ. Comenian. (N.S.) 76 (2007), no. 1, 77-83. MR.2331055

[14] Bernd Kawohl, Variations on the p-Laplacian, Nonlinear elliptic partial differential equations, Contemp. Math., vol. 540, Amer. Math. Soc., Providence, RI, 2011, pp. 35-46. MR2807407

[15] Bernd Kawohl, Stefan Krömer, and Jannis Kurtz, Radial eigenfunctions for the gametheoretic p-Laplacian on a ball, Differential Integral Equations 27 (2014), no. 7-8, 659-670. MR 3200758

[16] Bernd Kawohl and Jiří Horák, On the geometry of the p-Laplacian operator, Discrete Contin. Dyn. Syst. Ser. S 10 (2017), no. 4, 799-813. MR.3640538

[17] Kühn, M., On viscosity solutions and the normalized $p$-Laplacian, PhD thesis, University of Cologne, 2017

[18] Guozhen $\mathrm{Lu}$ and Peiyong Wang, A uniqueness theorem for degenerate elliptic equations, Geometric methods in PDE's, Lect. Notes Semin. Interdiscip. Mat., vol. 7, Semin. Interdiscip. Mat. (S.I.M.), Potenza, 2008, pp. 207-222. MR2605157

[19] Emmanouil Milakis and Luis E. Silvestre, Regularity for fully nonlinear elliptic equations with Neumann boundary data, Comm. Partial Differential Equations 31 (2006), no. 7-9, 1227-1252. MR 2254613

[20] Mikko Parviainen and Eero Ruosteenoja, Local regularity for time-dependent tug-of-war games with varying probabilities, J. Differential Equations 261 (2016), no. 2, 1357-1398. MR 3494400

[21] James Serrin, A symmetry problem in potential theory, Arch. Rational Mech. Anal. 43 (1971), 304-318. MR0333220

[22] Luis Silvestre and Boyan Sirakov, Boundary regularity for viscosity solutions of fully nonlinear elliptic equations, Comm. Partial Differential Equations 39 (2014), no. 9, 1694-1717. MR3246039

[23] Andrew L. Vogel, Symmetry and regularity for general regions having a solution to certain overdetermined boundary value problems, Atti Sem. Mat. Fis. Univ. Modena 40 (1992), no. 2, 443-484. MR 1200301

[24] H. F. Weinberger, Remark on the preceding paper of Serrin, Arch. Rational Mech. Anal. 43 (1971), 319-320. MR0333221

TIFR CAM, BANGALORE -560065, INDIA

Email address: agnidban@gmail.com

Mathematisches Institut, Universität Zu Köln, D-50923 Köln, Germany

Email address: kawohl@mi.uni-koeln.de 